\title{
Producción tecnológica latinoamericana con mayor visibilidad internacional: 1996-2007. Un estudio de caso: Brasil
}

\author{
Maidelyn Díaz Pérez*, Soleidy Rivero Amador ${ }^{\star \star}$, Félix de Moya-Anegón*
}

Resumen: La investigación propone la aplicación de un grupo de indicadores al documento de patente, lo que permite representar y analizar el comportamiento de la producción tecnológica existente en un determinado dominio. La propuesta se valida mediante un caso de estudio y los datos se procesan con una herramienta propia diseñada para realizar estudios patentométricos. Se representa el conocimiento tecnológico Latinoamericano publicado en Estados Unidos, entre septiembre de 1996 y abril del 2007, profundizando en el análisis del caso de Brasil como mayor productor de patentes. La batería de indicadores patentométricos que se proponen permiten cuantificar y cualificar el comportamiento de la producción tecnológica en términos de patentes concedidas. Se representan, a partir de las patentes analizadas, las capacidades tecnológicas en las áreas técnicas de aplicación, el posicionamiento de los institutos, centros de investigación, industrias, etc., así como la productividad de sus principales tecnólogos y relaciones entre ellos. Este último elemento de análisis favorece la cooperación y el intercambio tecnológico, así como la identificación de las prioridades y oportunidades. Todo lo cual, además, permite ayudar a planificar, implementar y evaluar políticas de investigación y desarrollo tecnológico a cualquier nivel, entre otras ventajas. Este tipo de análisis es considerado una herramienta indispensable para el mejor conocimiento de los recursos tecnológicos regionales, a la vez que apoya un aprovechamiento más eficiente de las capacidades tecnológicas existentes.

Palabras clave: Indicadores patentométricos, patentes, sistemas de análisis de información de patentes, producción tecnológica de América Latina, patentes de Brasil.

\section{Latin American technological production of greatest international visibility: 1996-2007. A case study: Brasil}

Abstract: This work proposes a set of indicators based on patent documents that allow researchers to represent and analyse the behaviour of technological production within a particular domain. This proposal is here validated via a case study. Collected data were processed employing an in-house tool designed for use in patentometric studies.

* Coordinadora Grupo de Gestión de Información y Conocimiento (PROGINTEC) . Universidad de Pinar del Río, Cuba. Correo-e: maidelyn@ict.upr.edu.cu.

** Departamento de Economía Global. Facultad de Ciencias Económicas. Universidad de Pinar del Río, Cuba. Correo-e: soly@eco.upr.edu.cu.

*** Consejo Superior de Investigaciones Científicas de España. Correo-e: felix.demoya@cchs. csic.es

Recibido: 20-1-09; 2. ${ }^{a}$ versión: 27-7-09; 3. ${ }^{a}$ version: 11-9-09; aceptado: 24-9-09. 
Latin American technological knowledge published in the USA between September 1996 and April 1997 was examined, paying special attention to that of Brazil, the largest Latin American filer of patents. The set of patentometric indicators proposed allows one to quantify and qualify the behaviour of technological production in terms of the patents granted. Using the patents analysed, the technological capacity revealed in the different technical areas covered is represented, along with the ranking of institutes, research centres and industries, etc. The productivity of their principal technologists and their relationships is also shown. The latter element of analysis favours cooperation and technological exchange, as well as the identification of priorities and opportunities. The above information is an aid for the planning, implementation and assessment of research and technological development policies at any level. This kind of analysis is considered an indispensable tool for improving our knowledge of regional technological resources. It also encourages the better use of existing technological capacities.

Keywords: Patentometric indicators, patents, patent information, analysis systems, Latin American technological production, Brazilian patents.

\section{Introducción}

Uno de los caminos que permite la supervivencia de una organización, en el actual mundo globalizado y competitivo, es el impulso a la innovación tecnológica. La gestión de la innovación tecnológica requiere de la puesta en marcha de un conjunto de mecanismos, entre los que se encuentra la protección de la propiedad intelectual. Las patentes, licencias, derechos de autor, marcas y secretos comerciales, entre otras figuras, forman un conjunto de herramientas legales, a través de las cuales, autores, inventores e innovadores buscan el cumplimiento, de su legítimo derecho sobre sus resultados.

Un inventor, al disfrutar de derechos exclusivos por un período determinado, puede recuperar lo que ha invertido y redimir los costes por concepto de I+D. Del mismo modo, el sistema de patentes es una forma de potenciar las inversiones encaminadas a promover y comercializar nuevas invenciones para que las personas puedan disfrutar de los resultados de la innovación (Díaz, 2005). Estos sistemas persiguen difundir el conocimiento y la información entre el público mediante la publicación de las solicitudes de patente y las patentes otorgadas. Y a su vez, la publicación del documento permite realizar análisis con la información contenida en las patentes.

Los análisis de patentes favorecen el desarrollo de diversos estudios, tanto en el sector académico, como en la investigación, la empresa o la industria. Dentro de los cuales se encuentran: conocer el estado del arte de la tecnología; buscar soluciones a problemas tecnológicos e innovadores; identificar socios, tendencias tecnológicas y tecnologías adquiribles; elaborar perfiles tecnológicos de productos y empresas; apoyar y orientar programas de I+D; proteger los resultados alcanzados en la investigación y desarrollo de la organización y/o el país, etc. (Sánchez, et al., 2007). Sin embargo, aún se considera que la utilización de patentes para la toma de decisiones todavía no es un instrumento común en todos 
los frentes de investigación innovadores (Moya-Anegón y Díaz, 2008). La protección por patentes, es un proceso internacional influenciado por distintos factores, que van desde las normas internacionales y los procesos político-económicos hasta las particulares específicas del proceso de ciencia y técnica de cada nación.

En América Latina (AL) los organismos públicos y entidades del estado encargados de realizar los estudios con la información de patentes, utilizan generalmente como fuentes de información o datos, a las Oficinas Nacionales de Patentes de cada país. En parte, esto se justifica, porque lo que se ha medido, con mayor incidencia, hasta el presente, es la producción de cada país en relación con la cantidad de patentes de otros. Buscando principalmente indicadores cuantitativos en términos de patentes concedidas y solicitadas por nacionales y extranjeros. Donde para estos fines, las oficinas nacionales han sido, hasta el presente, el mejor referente.

Existen algunas limitaciones considerables de este documento para la comparación internacional entre países, como por ejemplo: diferencias entre los sistemas y oficinas de patentes de los diferentes países; discrepancias en la exigencia de criterios usados para la patentabilidad; desniveles de las diferentes economías, etc. Sin embargo, se opina que estos argumentos utilizados durante décadas en los estudios de patentes y que orientan la utilización de unas escasas estadísticas, están creando serias limitaciones y barreras a la hora de analizar, comprender e interpretar el conocimiento tecnológico disponible en forma de patente; arrojando los análisis de los países latinoamericanos a un contexto muy regional (Díaz, 2007).

Aunque se use el número de patentes solicitadas por terceros países en Oficinas Internacionales como indicador de actividad de innovación, junto a otros de corte económico, se presume en este estudio, que no son explotados a plenitud todos los posibles indicadores a aplicar, ni valoradas todas sus posibles interpretaciones; para representar, describir y comprender el desarrollo tecnológico e innovativo publicado en las patentes de los diferentes países (Díaz, 2007). Situación que ha prejuiciado significativamente la relevancia y visibilidad de la producción y el desarrollo tecnológico latinoamericanos a nivel internacional.

En la actualidad, más allá de las estadísticas que realiza la Organización Mundial de la Propiedad Intelectual (OMPI) cada año, y los datos que publica en su Informe Mundial sobre el Estado de la Ciencia, editado anualmente por la Red Iberoamericana de Indicadores de Ciencia y Tecnología (RICYT), no se disponen de otros informes por parte de organismo públicos. Estos desconocen, o no analizan: el comportamiento de la colaboración tecnológica entre países, institutos de investigación, tecnólogos, sectores técnicos, etc. (Díaz, 2007). Todos los estudios en este sentido provienen de la literatura científica, con casos de estudios muy específicos.

El grupo CINDOC, por ejemplo, es uno de los que más ha tratado de representar la realidad tecnológica de AL, realizando estudios específicos de países de la región con diferentes indicadores bibliométricos (Fernández, 2004; Plaza y Albert, 2001). Existen otros trabajos orientados al análisis métrico de la realidad científica y tecnológica latinoamericana, con estudios específicos en determinadas 
áreas. Por ejemplo, en el trabajo de Saavedra, 2002, se analiza bibliométricamente la producción científica en América Latina y el Caribe en Ciencias Agrícolas. La investigación de Alfaraz y Calviño, 2004, representa un análisis bibliométrico de la producción científica en Ciencia y Tecnología de los alimentos para el período 1991-2000 en Iberoamérica. Y el trabajo de Arenas, 2004, analiza la investigación Agrícola en América Latina y el Caribe buscando identificar las fortalezas y debilidades en la región. Además de otros trabajos muy interesantes como los de: Russell, 1987; Moya-Anegón y Herrero-Solana, 1999; Moya-Anegón y HerreroSolana, 2002; Herrero-Solana y Ríos-Gómez, 2006. Todos estos estudios constituyen antecedentes importantes de esta investigación, pero lo más importante es que se constata y confirma la importancia de aplicar indicadores bibliométricos a la documentación de patentes (Moya-Anegón y Díaz, 2008).

La colaboración internacional ha sido otro elemento de análisis en el contexto de América Latina, investigado por diferentes autores (Russell y Narváez, 1992; Narváez, 1993; Narváez, 1995; Sancho, 2006). La Asociación Latino-Iberoamericana de Gestión Tecnológica (ALTEC) es otra de las organizaciones regionales que se ha ocupado también del ejercicio de la gestión tecnológica en AL.

A pesar de estos referentes, lo cierto es que no abundan análisis que describan ampliamente la producción científico tecnológica latinoamericana. Y menos que utilicen diferentes tipos de indicadores y algoritmos de visualización para representar la producción de patentes de AL en EE.UU. Argumentos que pueden justificar el limitado estudio que las organizaciones internacionales hacen con la información de patente de los países latinoamericanos, reduciéndola a unas pocas cifras y comparaciones internacionales de datos (Díaz, 2007). Obviando que las patentes se definen dentro de un contexto legal y un escenario socioeconómico que les dota de significado y contenido, y de los que no se pueden desvincular (Sánchez, s.a).

En el caso específico de Brasil dentro del contexto latinoamericano, es oportuno comentar, que es el país más extenso de América del Sur y el quinto del mundo. Tiene límites con todos los países sudamericanos, exceptuando a Chile y Ecuador. Es una República Federativa (con 8,5 millones de kilómetros cuadrados de superficie) posee 26 Estados y el Distrito Federal, cinco regiones geopolíticas heterogéneas y 5.508 municipalidades. Este país latinoamericano tiene una población total estimada en 169.544.443 habitantes, de la cual el 81,2\% se encuentra concentrada en áreas urbanas.

En Brasil se sitúan instituciones respetadas internacionalmente, como el Instituto Tecnológico da Aeronáutica, la Fundación Getúlio Vargas y la Fundación Oswaldo Cruz, la Universidad de São Paulo, reconocida en Iberoamérica y la Agencia Espacial Brasileña, que es la única institución iberoamericana que se integra en la Estación Espacial Internacional.

Desde la década de los noventa, Brasil, ha estado ocupando posiciones ventajosas - con respecto a países de América Latina- en diversos estudios relacionados con el comportamiento de las patentes. Acontecimiento que se ve reflejado en la literatura en autores tales como: Edsberg, 2000; Albuquerque, 2001; 
Matías-Pereira, 2004; Sánchez, 2007; Moya-Anegón y Herrero-Solana, 2002; LuisPinto y Moreira, 2005; Corder, 2006), entre otros. Otros estudios analizan la influencia de las patentes en el desarrollo económico (Morgado, 2005; Sikasso 2007; Luna, 2007). Y otros análisis se centran en el sector químico, específicamente en los medicamentos, siendo la biotecnología el más representativo (Antunes, 2001; Ferreira, 2007; Rovira, 2008).

A partir de estos antecedentes, la investigación tiene como objetivo representar la producción tecnológica de AL, en términos de patentes concedidas en EE.UU.; profundizando en el caso de Brasil. Proponiendo la utilización de diferentes indicadores simples y relacionales, así como diferentes formas de visualización de la información.

El presente estudio asume en primera instancia, a las patentes concedidas como indicador confiable para describir y representar el conocimiento tecnológico constituido, visible y con una realidad de mercado en AL. Se considera que, mayoritariamente, todas las invenciones científicas o tecnológicas de cualquier país de AL con posibilidades de explotación económica, solicitan patente en EE.UU. para su concesión.

En segunda instancia, asume que las patentes de AL concedidas en la Oficina de Patentes de Estados Unidos (USPTO), son las de mayor interés comercial y mayor visibilidad internacional, considerándolas como las más apropiadas y confiables, según los intereses del presente estudio.

Notables investigadores consideran que la Base de Datos (BDs) de los EE. UU. probablemente sea una de las fuentes de datos de patentes más fiables del mundo, junto a ESPACENET. Dado el rigor y alcance de su legislación sobre patentes; el grado de detalle y accesibilidad de los datos de patentes, además de la atracción técnica y sofisticado mercado que poseen (Pavitt, 1988). Además de permitir análisis con la tecnología desde su comunicación con las ciencias (Narin y Hamilton, 1996; Narin, 1997). Todo lo cual ampara su amplia utilización por disímiles investigadores del tema.

\section{Métodos y procedimientos}

\subsection{Elección de la fuente de datos}

Se selecciona la USPTO, entre otros argumentos por:

- ser una de las oficinas y una de las tres BDs de patentes, más importantes del mundo (Michel y Bernd, 2001), junto a la BDs de la Oficina Europea de Patentes (EPO) y la Organización de Información Japonesa de Patentes (JAPIO) (las tres juntas procesan el 86\% de todas las solicitudes de patentes del mundo) (Trilateral Co-operation, 2005);

- por tener un desfase significativo con la Unión Europea (UE), respecto al número de solicitudes de patentes que se presentan cada año (Anthony Arundel, de MERIT Universidad de Maastricht, comenta que una parte im- 
portante del desfase en las patentes de alta tecnología entre la UE y los EE.UU. puede explicarse por la gran concentración de industrias estadounidense en sectores de alta tecnología además de proyectar una estrategia de patentamiento de carácter defensivo);

- por encontrarse geográficamente en el continente americano (el estudio al estar dirigido a $\mathrm{AL}$, supone por razones económicas, comerciales, políticas, etc., que las oficinas nacionales y la de EE.UU. sean las más factibles para sus protecciones, encontrándose las patentes de mayor relevancia tecnológica en la USPTO);

- por presentar una significativa cobertura internacional;

- por publicar íntegramente el texto de la patente a diferencia de otras bases de datos del mundo, etc.

\subsection{Estrategia de búsqueda: América Latina}

Interrogar a la BDs de la USPTO sobre los registros de patentes concedidos entre el 1 de septiembre del año 1996 y el 17 de abril del 2007. Cuyo país del inventor (ICN) y país del titular (ACN) (query independiente en cada caso) procedan de alguno de los 21 países de AL seleccionados para el estudio (Anexo 1). Los datos son procesados íntegramente en todas sus fases, desde la descarga hasta la visualización, por el software PROINTEC, herramienta diseñada y desarrollada por el Grupo de Gestión de Información y Conocimiento (PROGINTEC) de la Universidad de Pinar del Río en Cuba.

\section{Query AL por país del inventor:}

$\mathrm{PD} / 9 / 1 / 1996>4 / 17 / 2007$ and (ICN/AR or ICN/BO or ICN/BR or ICN/CL or ICN/CO or ICN/CU or ICN/CR or ICN/EC or ICN/SV or ICN/GT or ICN/HN or $\mathrm{ICN} / \mathrm{MX}$ or ICN/NI or ICN/PA or ICN/PY or ICN/PE or ICN/DO or ICN/UY or $\mathrm{ICN} / \mathrm{VE}$ or ICN/PE or ICN/MQ or ICN/HT)

\section{Query AL por país del titular:}

$\mathrm{PD} / 9 / 1 / 1996>4 / 17 / 2007$ and (ACN/AR or ACN/BO or ACN/BR or ACN/CL or $\mathrm{ACN} / \mathrm{CO}$ or $\mathrm{ACN} / \mathrm{CU}$ or $\mathrm{ACN} / \mathrm{CR}$ or $\mathrm{ACN} / \mathrm{EC}$ or $\mathrm{ACN} / \mathrm{SV}$ or $\mathrm{ACN} / \mathrm{GT}$ or $\mathrm{ACN} / \mathrm{HN}$ or $\mathrm{ACN} / \mathrm{MX}$ or $\mathrm{ACN} / \mathrm{NI}$ or $\mathrm{ACN} / \mathrm{PA}$ or $\mathrm{ACN} / \mathrm{PY}$ or $\mathrm{ACN} / \mathrm{PE}$ or $\mathrm{ACN} / \mathrm{DO}$ or $\mathrm{ACN} /$ UY or ACN/VE or ACN/MQ or CAN/HT)

En el caso de Brasil se determinó buscar por país del titular (de forma independiente), restringiendo la búsqueda sólo a la patentes con titularidad en este país. Manteniendo el mismo período de tiempo, entre septiembre de 1996 y abril del 2007. Mientras el comportamiento de los principales inventores brasileños, esta incluido en el estudio general de América Latina.

\section{Query Brasil por país del titular:}

$\mathrm{PD} / 9 / 1 / 1996>4 / 17 / 2007$ and (ACN/BR). 


\section{Medición: indicadores}

Se propone un grupo de indicadores de patentes, cuya base es principalmente cuantitativa, establecida por recuentos estadísticos y conteos de frecuencia. Además de introducir un significativo número de indicadores relacionales que analizan en un mismo grafo varias variables bajo estudio, aplicando técnicas métricas.

\section{Aplicación de indicadores: Caso de Estudio}

$\mathrm{Al}$ analizar la cantidad de patentes por país del titular donde los titulares procedan de cualquiera de los 21 países de AL seleccionados para un primer análisis (Tabla I), se obtiene que: Paraguay, Martinica y Haití no aparecen representados. Lo que indica que no poseen titularidad de patentes en EE.UU. desde hace más de diez años. Mientras Brasil, México, Venezuela y Argentina son los prin-

\section{TABLA I}

Patentes de AL distribuidas por años

\begin{tabular}{l|r|r|r|r|r|r|r|r|r|r|r|r|r}
\hline \multicolumn{1}{c|}{ Países (Tit)/Años } & $\mathbf{1 9 9 6}$ & $\mathbf{1 9 9 7}$ & $\mathbf{1 9 9 8}$ & $\mathbf{1 9 9 9}$ & $\mathbf{2 0 0 0}$ & $\mathbf{2 0 0 1}$ & $\mathbf{2 0 0 2}$ & $\mathbf{2 0 0 3}$ & $\mathbf{2 0 0 4}$ & $\mathbf{2 0 0 5}$ & $\mathbf{2 0 0 6}$ & $\mathbf{2 0 0 7}$ & Total \\
\hline Argentina & 4 & 9 & 9 & 6 & 15 & 8 & 8 & 16 & 13 & 5 & 12 & 3 & 108 \\
\hline Bolivia & 17 & 23 & 51 & 58 & 49 & 59 & 58 & 129 & 108 & 45 & 67 & 15 & 679 \\
\hline Brasil & & 1 & 7 & 5 & 7 & 7 & 4 & 7 & 5 & 6 & 6 & 3 & 58 \\
\hline Chile & & 1 & & 5 & & 4 & 4 & & 4 & 1 & 3 & & 22 \\
\hline Colombia & & 1 & & 3 & 1 & 2 & 2 & & 2 & 9 & 12 & & 32 \\
\hline Costa Rica & & 5 & 7 & 4 & 5 & 4 & 10 & 6 & 2 & 3 & 2 & 1 & 49 \\
\hline Cuba & & & & & & & & & & & 1 & & 1 \\
\hline Respub. Dominicana & & & & 1 & & 1 & & & & & & & 2 \\
\hline Ecuador & & & 1 & 2 & & & 5 & & 1 & & & & 9 \\
\hline El Salvador & & 1 & & 1 & & & & 1 & & 1 & & & 4 \\
\hline Guatemala & 12 & 12 & 30 & 39 & 34 & 36 & 39 & 36 & 24 & 16 & 17 & 7 & 302 \\
\hline Honduras & & & & & & & & & & & & & \\
\hline
\end{tabular}

Fuente: Software proINTEC. 
cipales países de AL introduciendo tecnologías en EE.UU. para su protección, seguidos por Chile, Panamá y Cuba, con cifras no tan demostrativas, pero igual de relevantes, al considerar el tamaño y número de habitantes respecto a los anteriores. En total existen 1482 patentes de AL registradas en EE.UU. en el ciclo de tiempo que se analiza.

Dentro de los países estudiados, el caso de Brasil es el más significativo, al ostentar la mayor cifra de patentes de AL, con un total de 679 patentes concedidas en la USPTO. La Figura 1 muestra el comportamiento por años de esta actividad en Brasil, destacándose los años 2003 y 2004 como los de mayor concesión de patentes. Existiendo un promedio de aproximadamente 60 patentes concedidas por año. En el análisis por ciudad del titular, el sistema filtró las ciudades donde residen los comercializadores de las tecnologías producidas por Brasil. Constituyendo Sao Paulo y Río de Janeiro las regiones geográficas más tecnólogas y con mayores intereses comerciales introducidos en EE.UU. Información de interés que puede ser utilizada para desarrollar proyectos, alianzas estratégicas, licenciamientos, etc., con universidades, empresas, industrias, investigadores, tecnólogos, etc., de estas ciudades con alto potencial científico tecnológico en Brasil.

FIGURA 1

Número de patentes de Brasil

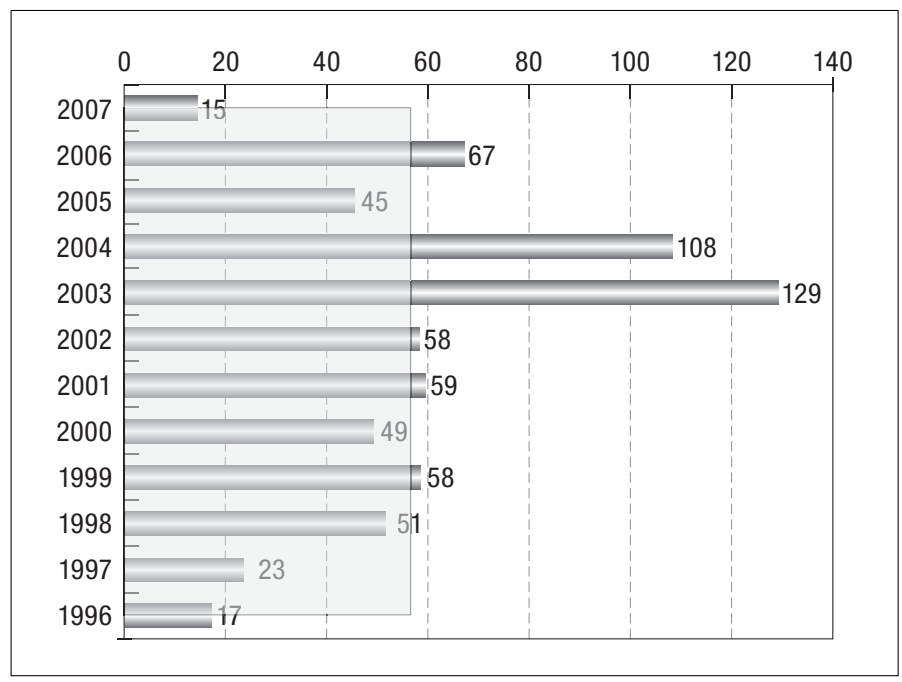

Fuente: Software proINTEC.

Para analizar la cantidad de patentes de titulares de AL por países, se restringió la búsqueda a los titulares con más de cuatro registros de invención (Tabla II). Mostrándose únicamente 8 países con titulares que cumplan esta condición. De 
los cuales ninguno de ellos posee más de 4 patentes registradas en otros países de $\mathrm{AL}$, de lo que se puede inferir que el desarrollo de las tecnologías en AL está muy regionalizado, en términos de dominio de mercados. Otro de los resultados interesantes tras aplicar este indicador fue descubrir que Venezuela ha logrado ocupar el tercer lugar por países en AL, principalmente con solo dos titulares comercializando de manera intensiva sus tecnologías en el mercado internacional. Igual que Argentina, que ocupa el cuarto lugar por países, con solo dos titulares con más de cuatro patentes, seguido de Cuba con dos titulares. Mientras que Brasil y México, en correspondencia con su lugar por países, ocupan los dos primeros lugares al poseer el mayor número de titulares en AL protegiendo las tecnologías introducidas en EE.UU.

TABLA II

Patentes de titulares de AL por países

\begin{tabular}{|c|c|c|c|c|c|c|c|c|}
\hline Titulares/País & 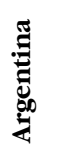 & :ृ & 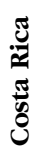 & $\frac{\pi}{3}$ & 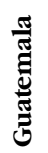 & 恖 & 莺 & 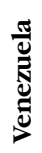 \\
\hline Acertek, S.A. de C.V. & & & & & & 5 & & \\
\hline Ballado Investments Inc. & & & & & & & 9 & \\
\hline Brasilata S.A. Embalagens Metalicas & & 7 & & & & & & \\
\hline $\begin{array}{l}\text { Centro de Ingeniería Genética y Biotecno- } \\
\text { logía }\end{array}$ & & & & 14 & & & & \\
\hline Centro de Inmunologia Molecular (CIM) & & & & 12 & & & & \\
\hline Col-Ven S.A. & 8 & & & & & & & \\
\hline Comercial Acros Whirlpool, S.A. de C.V. & & & & & & 7 & & \\
\hline Companhia Vale Do Rio Doce & & 10 & & & & & & \\
\hline Dana Industrial S/A & & 12 & & & & & & \\
\hline $\begin{array}{l}\text { Empresa Brasileira de Compressores S.A.- } \\
\text { Embraco }\end{array}$ & & 50 & & & & & & \\
\hline Fábricas Monterrey, S.A. de C.V. & & & & & & 5 & & \\
\hline Forjas Taurus S.A. & & 7 & & & & & & \\
\hline Fundaçao Oswaldo Cruz-Fiocruz & & 7 & & & & & & \\
\hline $\begin{array}{l}\text { Fundaçao de Amparo a Pesquisa do Estado } \\
\text { de Sao Paulo }\end{array}$ & & 6 & & & & & & \\
\hline Grendene S.A. & & 29 & & & & & & \\
\hline Hylsa, S.A. de C.V. & & & & & & 15 & & \\
\hline
\end{tabular}


TABLA II (continuación)

\begin{tabular}{|c|c|c|c|c|c|c|c|c|}
\hline Titulares/País & 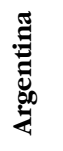 & 苞 & 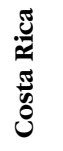 & $\frac{0}{3}$ & 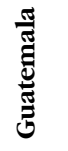 & 恖 & 莺 & 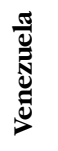 \\
\hline $\begin{array}{l}\text { Industria e Comercio de Cosmeticos Natu- } \\
\text { ra Ltda }\end{array}$ & & 12 & & & & & & \\
\hline Instituto Mexicano del Petróleo & & & & & & 5 & & \\
\hline Intevep, S.A. & & & & & & & & 118 \\
\hline $\begin{array}{l}\text { Johnson \& Johnson Industria \& Comercio, } \\
\text { LTDA }\end{array}$ & & 35 & & & & & & \\
\hline Koblenz Electrica, S.A. de C.V. & & & & & & 8 & & \\
\hline Laboratorios Dalmer SA & & & & 5 & & & & \\
\hline $\begin{array}{l}\text { Magic Toys do Brasil Industria e Comercio } \\
\text { LTDA }\end{array}$ & & 5 & & & & & & \\
\hline Maquinas Agricolas Jacto S.A. & & 6 & & & & & & \\
\hline Metagal Industria e Commercio LTDA & & 18 & & & & & & \\
\hline Metal Leve S.A. Industria e Comercio & & 5 & & & & & & \\
\hline Metalgrafica Rojek Ltda. & & 5 & & & & & & \\
\hline Multibras S.A. Electrodomesticos & & 35 & & & & & & \\
\hline Petroleo Brasileiro S.A.-Petrobras & & 98 & & & & & & \\
\hline Polimeros Industriales C.A. & & & & & & & & 6 \\
\hline Prolec GE, S. de R.L. de C.V. & & & & & & 5 & & \\
\hline Roberto Gonzalez Barrera & & & & & & 6 & & \\
\hline Servicios Condumex S.A. de C.V. & & & & & & 14 & & \\
\hline Siderca S.A.I.C. & 8 & & & & & & & \\
\hline Tenedora Nemak S.A. de C.V. & & & & & & 5 & & \\
\hline Transmisiones TSP, S.A. de C.V. & & & & & & 7 & & \\
\hline Universidad Nacional Autonoma de Mexico & & & & & & 7 & & \\
\hline Universidade Federal de Minas Gerais & & 6 & & & & & & \\
\hline Vidriera Monterrey, S.A. de C.V. & & & & & & 5 & & \\
\hline Vidrio Plano de Mexico, S.A. De C.V. & & & & & & 5 & & \\
\hline Visio Plant & & & 17 & & & & & \\
\hline Wahler Metalurgica Ltda. & & 9 & & & & & & \\
\hline Xanadu Candle International, Limited & & & & & 5 & & & \\
\hline
\end{tabular}


El resto de los países tienen titulares con una productividad discreta, generalmente con tan solo uno o dos titulares con más de tres patentes en un año, e incluso, existen más de diez países de los estudiados que no se representan en esta tabla, al presentar poca productividad por años y no cumplir con los criterios de poda establecidos en la búsqueda. Información que puede ser interpretada de diferentes formas según los objetivos de sus posibles análisis.

$\mathrm{Al}$ analizar la productividad de los titulares (industrias, empresas, universidades, etc.) (Tabla III) se observa que la firma Intevep, S.A. (Instituto de Tecnología Venezolana para el Petróleo) muestra una presencia sostenida en el mercado en todos los años estudiados, con cifras significativas de patentes concedidas en los años 1999, 2002 y 2000. Lo que en general permite deducir, un fuerte dominio en las tecnologías que produce e introduce al mercado internacional de forma mantenida cada año. Lo que se justifica al ser la filial de Investigación y Desarrollo de Petróleos de Venezuela, S.A. (PDVSA), considera el brazo tecnológico de la corporación.

\section{TABLA III}

Titulares de AL con más de 4 patentes

\begin{tabular}{|c|c|c|c|c|c|c|c|c|c|c|c|c|}
\hline Titulares/Años & 1997 & 1998 & 1999 & 2000 & 2001 & 2002 & 2003 & 2004 & 2005 & 2006 & 2007 & Total \\
\hline Cerveceria Polar, C.A. & & & & & & 2 & & & & & & 2 \\
\hline Deltaven, S.A. & & & & & & & & 2 & & & & 2 \\
\hline Industrias Venoco C.A. & & & & & & 1 & 1 & & & & & 2 \\
\hline Inelectra S.A. & & & & & & & & 1 & & & & 1 \\
\hline $\begin{array}{l}\text { International Briquettes } \\
\text { Holding }\end{array}$ & & & & 1 & & & & & & & & 1 \\
\hline Intevep, S.A. & 8 & 19 & 25 & 12 & 8 & 13 & 10 & 8 & 3 & 6 & 2 & 114 \\
\hline Inversiones Bernoulli C.A. & & 1 & & & & & & & & & & 1 \\
\hline Minelli Modas, C.A. & & & & & & & 1 & & & & & 1 \\
\hline Multiprens C.A. & & & 1 & & 2 & 1 & & & & & & 4 \\
\hline Orinoco Iron, C.A. & & & & 2 & 1 & & & & & & & 3 \\
\hline Plastic-Envases, C.A. & & & & & 1 & & & & & & & 1 \\
\hline Polimeros Industriales C.A. & & & & 2 & & 1 & 1 & 2 & & & & 6 \\
\hline $\begin{array}{l}\text { Provecto Tecnico R-2000, } \\
\quad \text { Ca }\end{array}$ & & & & & & & & & 1 & & & 1 \\
\hline Universidad Simon Bolivar & & & 1 & 2 & & & & & & & & 3 \\
\hline Total & 8 & 20 & 27 & 19 & 12 & 18 & 13 & 13 & 4 & 6 & 2 & 142 \\
\hline
\end{tabular}


Otro comportamiento significativo en la actividad por titulares lo tiene Petrobras, empresa de energía brasileña, quien de igual forma refleja un fuerte dominio en sus producciones con una permanencia mantenida en el mercado en los últimos diez años. Lo que se justifica porque opera en forma activa en el mercado internacional del petróleo y en el intercambio de una importante diversidad de productos relacionados con la industria hidrocarburífera. Destacándose por utilizar alta tecnología en operaciones de exploración y producción de petróleo en aguas abiertas (off shore), contando con el récord de la planta de producción de crudo más profunda del mundo. Todo lo cuál exige la generación de tecnología propia, protegida mediante patentes.

Otro de los titulares importantes de AL es Embraco de Brasil. Empresa de compresores famosa en el mundo entero por la tecnología avanzada de sus productos, su oferta de soluciones innovadoras en refrigeración y su enfoque a la investigación. Actualmente invierte hasta un 3\% de sus ingresos netos anuales en investigación y desarrollo, junto a Multibras, empresa desarrolladora de electrodomésticos brasileños con cobertura internacional, a Dana Industrial, Metagal Industria y Johnson and Johnson, esta última que aunque no es titular latinoamericana, sus tecnologías tienen una fuerte presencia en los países de la región.

Los análisis por Clasificación Internacional de Patentes (CIP) según los indicadores que propone esta investigación, se pueden realizar en cualquier nivel de clasificación de la técnica (Anexo 2). Identificando en cada caso, tanto en AL (Tabla IV) en general, como en Brasil (Tabla V) en particular, cuáles son las secciones de técnica en las que más se han trabajado. Esta distribución de patentes por temáticas permite conocer la composición del portafolio de patentes de la región por países, y una primera aproximación a la estructuración de su conocimiento tecnológico.

La sección dedicada a la Química, Metalurgia (C) es la más trabajada en AL con un total de 250 patentes, seguida de Técnicas Industriales Diversas, Transporte (B) con 224 patentes. Y esta última sección igualmente es la más trabajada en Brasil con 103 concesiones, seguida por Mecánica, Iluminación, Calefacción, etc., con 85 invenciones concedidas a Brasil en esta área de la técnica (Tabla IV). Como dato significativo del resultado de la aplicación de este indicador, es dar a conocer a Brasil y México como los únicos países que investigan en todas las secciones de la técnica en AL.

En el análisis hasta el nivel de clase, en AL se obtiene que la clase temática más trabajada es la A61, perteneciente al área de las Ciencias Médicas o Veterinarias, e Higiene. La cual presenta 121 patentes, de un promedio de 25 patentes por años en cada clase en AL. Este estudio por clases permite conocer las especialidades de la técnica en la que se trabaja, las cuales no tienen que coincidir razonablemente con el estudio por secciones, contradicción que se presenta, por ejemplo, en este estudio.

La sección C y B presentan, en general, más patentes, pero la sección A presenta, en particular, más invenciones en especialidades relacionadas con sus temáticas. Análisis que permite adoptar diferentes conductas investigativas según 


\section{TABLA IV}

Titulares de AL por sección de la CIP

\begin{tabular}{|c|c|c|c|c|c|c|c|c|c|}
\hline Países (Tit)/CIP [sección] & $\mathbf{A}$ & B & C & $\mathbf{D}$ & $\mathbf{E}$ & $\mathbf{F}$ & G & $\mathbf{H}$ & Total \\
\hline Argentina & 33 & 18 & 16 & & 6 & 6 & 7 & 1 & 87 \\
\hline Brasil & 65 & 103 & 69 & 3 & 28 & 85 & 48 & 23 & 424 \\
\hline Chile & 8 & 6 & 14 & 1 & & 2 & 3 & 1 & 35 \\
\hline Colombia & 6 & 5 & 2 & & & 1 & 2 & & 16 \\
\hline Costa Rica & 10 & 1 & 4 & & 2 & 2 & & & 19 \\
\hline Cuba & 29 & & 25 & & & & 2 & & 56 \\
\hline República Dominicana & 1 & & & & & & & & 1 \\
\hline Ecuador & 1 & & & & & & & & 1 \\
\hline El Salvador & & & 1 & & & & & & 1 \\
\hline Guatemala & 1 & & 2 & & & & & & 3 \\
\hline México & 36 & 44 & 58 & 8 & 3 & 21 & 12 & 15 & 197 \\
\hline Nicaragua & & & & & & & 1 & & 1 \\
\hline Panamá & 8 & 12 & 5 & 1 & 1 & 3 & & 3 & 33 \\
\hline Perú & 1 & 1 & 1 & 1 & & 1 & 1 & & 6 \\
\hline Uruguay & 3 & & 1 & & & & 1 & & 5 \\
\hline Venezuela & 4 & 34 & 52 & & 6 & 6 & 9 & & 111 \\
\hline Total & 206 & 224 & 250 & 14 & 46 & 127 & 86 & 43 & 996 \\
\hline
\end{tabular}

Fuente: Software proINTEC.

TABLA V

Patentes de titulares de Brasil por sección de la CIP

\begin{tabular}{|c|c|c|c|c|c|c|c|}
\hline Titulares/CIP & $\mathbf{A}$ & B & C & $\mathbf{E}$ & $\mathbf{F}$ & G & $\mathbf{H}$ \\
\hline Dana Industrial S/A & & & & & 7 & & \\
\hline Empresa Brasileira de Compressores S.A.-Embraco & & & & & 18 & & 7 \\
\hline Fundacao de Amparo a Pesquisa do Estado de Sao Paulo & 5 & & & & & & \\
\hline Johnson \& Johnson Industria \& Comercio, LTDA & 7 & & & & & & \\
\hline Metagal Industria E Commercio LTDA & & & & & & 12 & \\
\hline Metalgrafica Rojek Ltda. & & 5 & & & & & \\
\hline Multibras S.A. Electrodomésticos & & & & & 10 & & \\
\hline Petroleo Brasileiro S.A.-Petrobras & & 34 & 16 & 22 & 8 & & \\
\hline Universidade Federal de Minas Gerais & & & 5 & & & & \\
\hline Wahler Metalúrgica Ltda. & & & & & 5 & & \\
\hline
\end{tabular}

Fuente: Software proINTEC. 
sea el caso; y hacer varias lecturas del conocimiento tecnológico disponible como patentes de invención.

En el análisis por el tercer nivel de la CIP, se filtraron las sub especialidades con más de cuatro patentes (Anexo 3). En el caso de la clase A61, se identificaron como las subclases más representativas: A61K (patentes destinadas a Preparaciones de uso medico, dental o para el aseo); A61F (patentes sobre filtros implantables en los vasos sanguíneos. prótesis. dispositivos que mantienen la luz o que evitan el colapso de estructuras tubulares, dispositivos de ortopedia, cura o para la contracepción, fomentación, tratamiento o protección de ojos y oídos, vendajes, apósitos o compresas absorbentes, botiquines de primeros auxilios); y A61B (patentes sobre diagnóstico, cirugía, identificación), etc.

La visualización de este indicador en la Figura 2, permite además ver los países que están investigando en las subclases temáticas más trabajadas. Destacándose la presencia de sólo cinco países, de 21 países muestreados, donde Brasil es quien más investiga en diferentes subclases de la técnica, seguido de México, Venezuela, Argentina y Cuba, respectivamente. Este último país con un número

FIGURA 2

Titulares de AL por subclases de la CIP

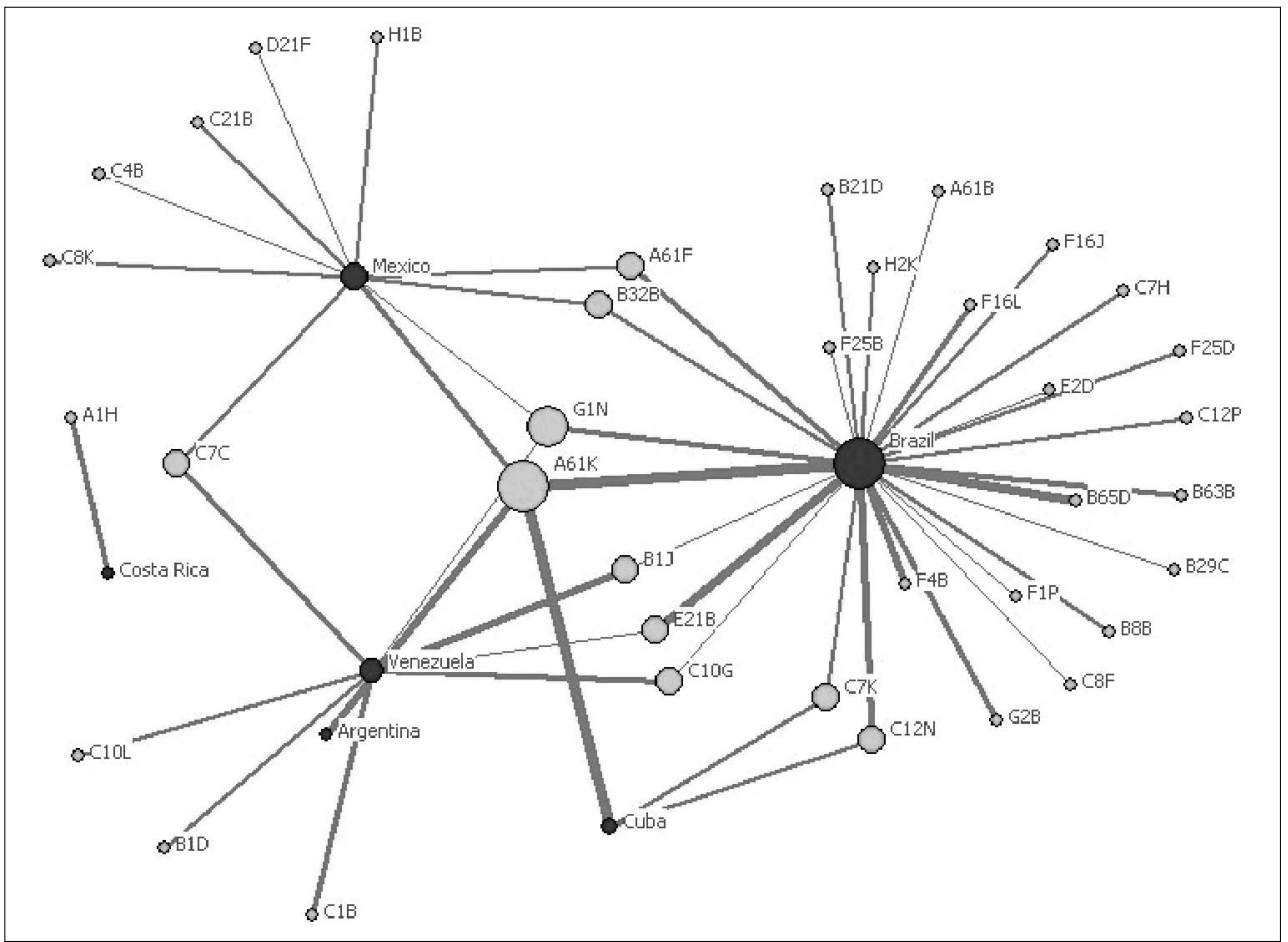

Fuente: Software proINTEC. 
significativo de patentes (solo superado por Brasil) en la subclase A61K, la clase técnica más trabajada en toda AL.

En un análisis de subclases por titulares se conoció, por ejemplo, que Intevep de Venezuela trabaja masivamente en las subclases C10G, C7C, C1B, e intensamente en la B1J. Esta última trabajada igualmente por Petróleo Brasilero, quién investiga de igual manera en la B63B y en mayor medida en la E21B. La aplicación de este indicador permite, en la medida que vaya efectuándose de forma más profunda, estructurar todo el conocimiento científico tecnológico por los diferentes sectores reconocidos de la técnica, incluso hasta de cada signatario latinoamericano en el contexto internacional.

Al realizar un análisis similar, de forma independiente con Brasil, se llega a conocer quienes están financiando las invenciones en Brasil y en que áreas del conocimiento técnico. La Tabla VI muestra los titulares de Brasil con más de 4 patentes por sección de la técnica. El análisis constata que Petrobras es la firma más emprendedora y la más transdisciplinar en el desarrollo de sus tecnologías, al patentar resultados en cuatro secciones temáticas diferentes para lograr la novedad, carácter inventivo y aplicación industrial de sus productos y tecnologías. Embraco tiene en total 25 patentes en el período estudiado, y trabaja potencialmente en dos secciones técnicas. Mientras el resto de las principales industrias, empresas, firmas y universidades de Brasil, tienen un perfil técnico definido en sus invenciones.

TABLA VI

Titulares de Brasil con más de 4 patentes por sección de la técnica.

\begin{tabular}{|c|c|c|c|c|c|c|c|c|}
\hline Titulares/CIP & $\mathbf{A}$ & $\mathbf{B}$ & C & $\mathbf{E}$ & $\mathbf{F}$ & G & $\mathbf{H}$ & Total \\
\hline Dana Industrial S/A & & & & & 7 & & & 7 \\
\hline Empresa Brasileira de Compressores S.A.-Embraco & & & & & 18 & & 7 & 25 \\
\hline $\begin{array}{l}\text { Fundaçao de Amparo a Pesquisa do Estado de Sao } \\
\text { Paulo }\end{array}$ & 5 & & & & & & & 5 \\
\hline Johnson \& Johnson Industria \& Comercio, LTDA & 7 & & & & & & & 7 \\
\hline Metagal Industria E Commercio LTDA & & & & & & 12 & & 12 \\
\hline Metalgrafica Rojek Ltda. & & 5 & & & & & & 5 \\
\hline Multibras S.A. Electrodomesticos & & & & & 10 & & & 10 \\
\hline Petroleo Brasileiro S.A.-Petrobras & & 34 & 16 & 22 & 8 & & & 80 \\
\hline Universidade Federal de Minas Gerais & & & 5 & & & & & 5 \\
\hline Wahler Metalurgica Ltda. & & & & & 5 & & & 5 \\
\hline Total & 12 & 39 & 21 & 22 & 48 & 12 & 7 & 161 \\
\hline
\end{tabular}


Otro aspecto que propone la investigación es analizar las clasificaciones conjuntas por subclases temáticas. La Figura 3 representa el caso de AL, evidenciando la veracidad de este indicador para obtener datos significativos de las relaciones e inter relaciones de los conocimientos estructurados en las diferentes áreas de la técnica. Las relaciones más novedosas se destacan entre especialidades de diferentes sectores, lo que genera la obteniendo de nuevas tecnologías y aplicaciones de productos, etc. Este tipo de análisis una vez interpretados por los especialistas de las diferentes ramas de la ciencia y la tecnología, arrojaran sustanciosos conocimientos respecto al estado del arte de muchas disciplinas científicas y sus interrelaciones tecnológicas.

\section{FIGURA 3}

\section{Clasificaciones conjuntas por subclases temáticas}

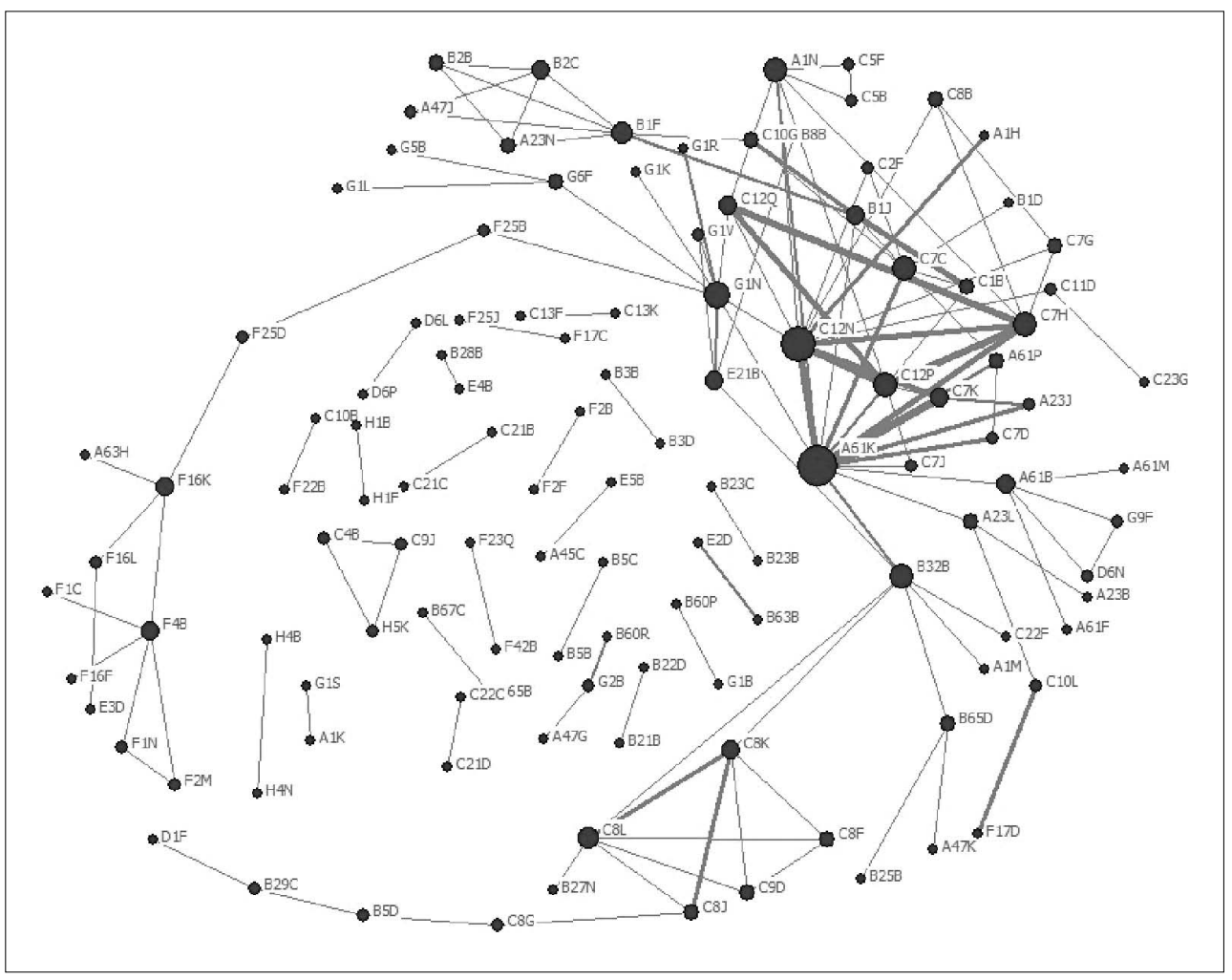

Fuente: Software proINTEC.

La Figura 4 muestra otro de los indicadores propuestos en esta investigación, visualizando las principales relaciones de titulación conjunta que se establecen entre secciones de la técnica. Por ejemplo, la C del sector Químico, Metalúrgico; 


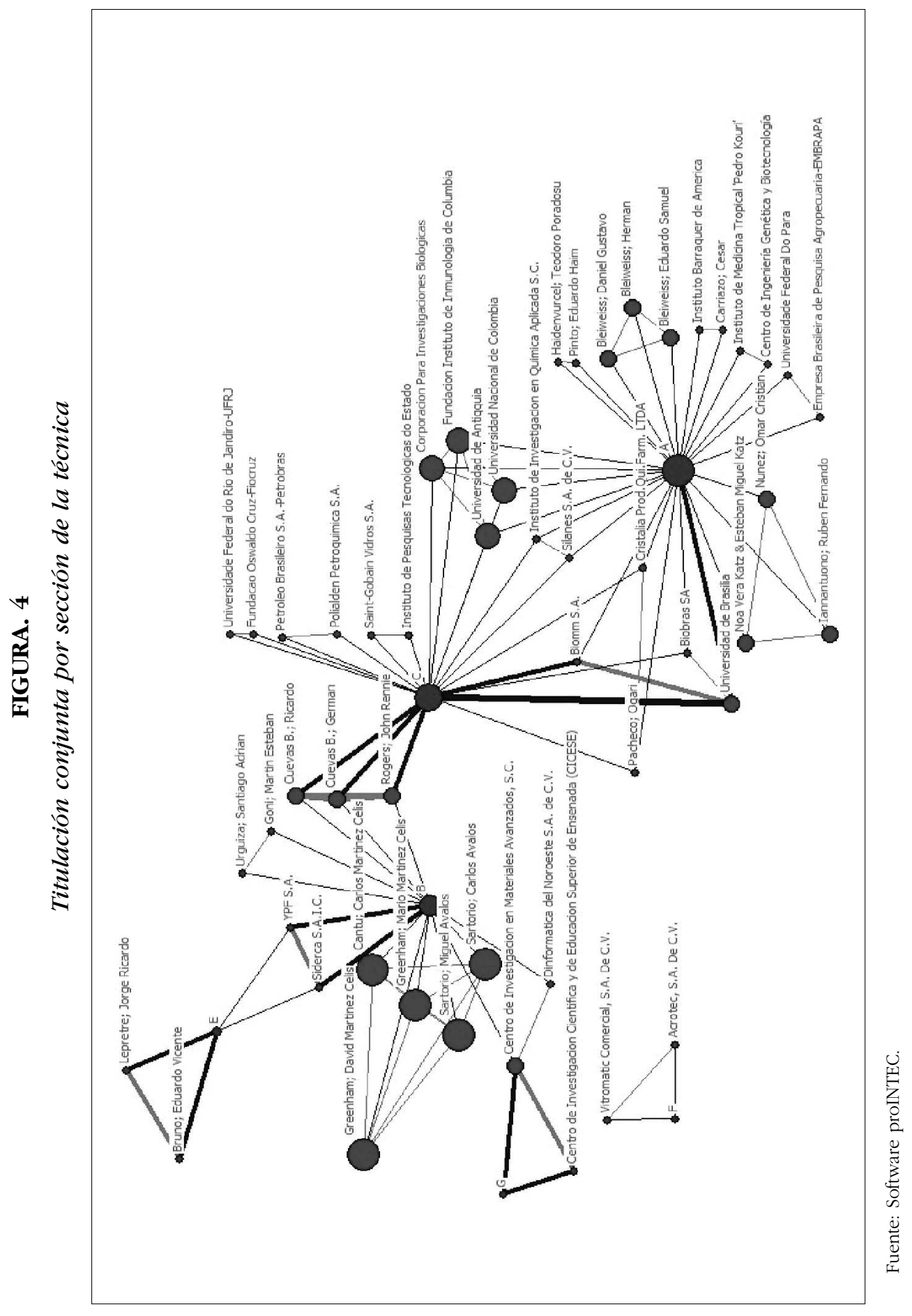


seguido del A Necesidades Corrientes de la Vida y del B sobre Técnicas Industriales Diversas, Transporte. Estas son las secciones técnicas que más titulación conjunta presentan en el tiempo que se analiza, coincidiendo con los sectores técnicos más comercializados por AL. Revelando, además, que las parejas de titulares que han trabajado en conjunto mayor cantidad de veces, son: Cuevas B. Ricardo, Cuervas B. German y Rogers John Renies. Seguidos de otras parejas como Biomm, S.A y la Universidad de Brasilia, entre otros. Pudiendo obtener a través de este indicador toda la representación de las relaciones de colaboración de los titulares por sección técnica de clasificación.

Y por último se aplicó un indicador que permite conocer como se comporta la colaboración entre países que originan la tecnología. Representando los países conjuntos que han trabajado en colaboración (Fig. 5), generando patentes bajo titulares latinoamericanos, en el período estudiado.

La Figura 5 muestra una rica estructura de relaciones entre inventores de diferentes países. Por ejemplo, una de las situaciones más significativas consiste en

\section{FIGURA 5}

\section{Colaboración entre países que originan la tecnología}

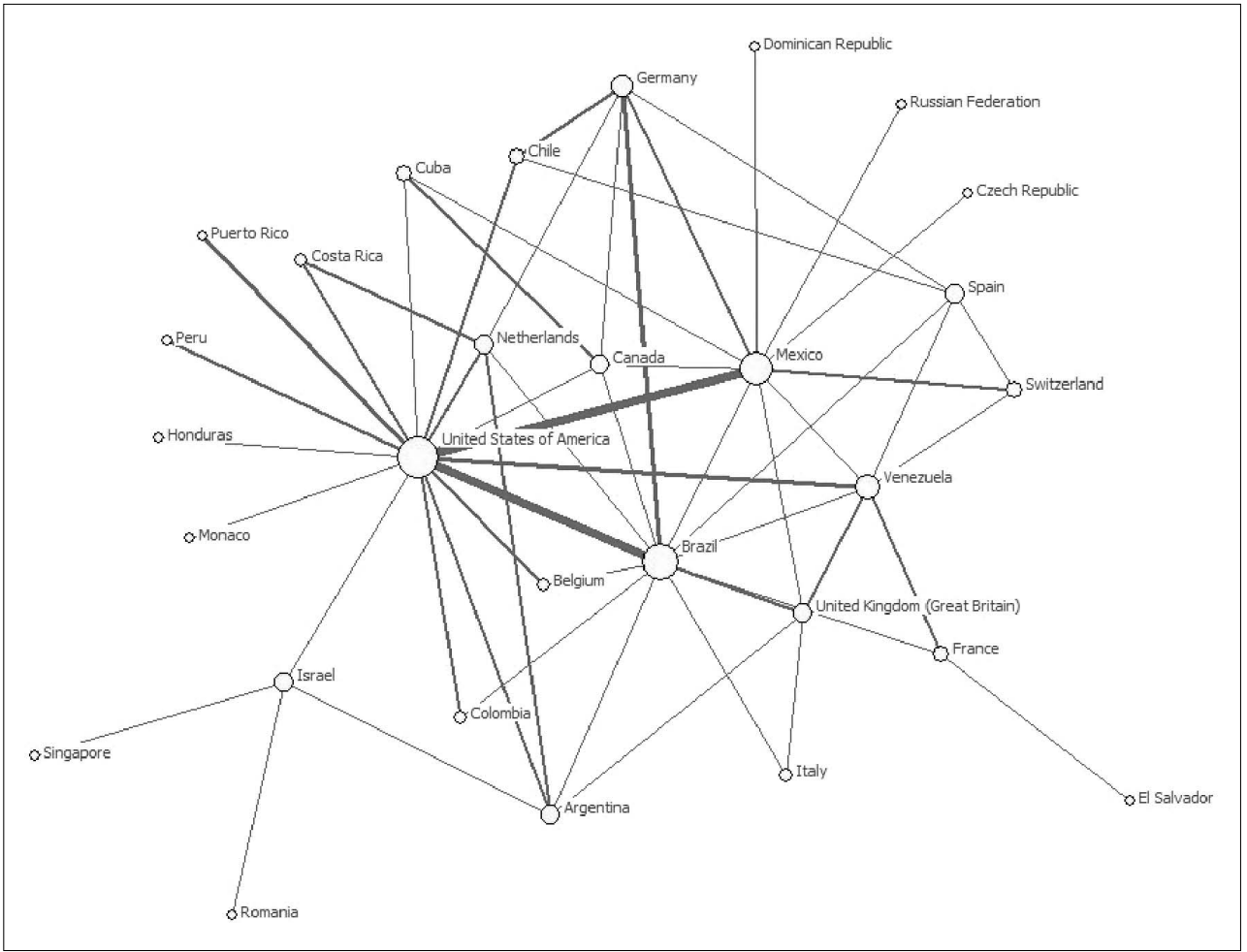

Fuente: Software proINTEC. 
la gran colaboración que existe entre inventores de varios países de AL con inventores de EE.UU., los que de forma conjunta tienen patentes bajo titulares latinoamericanos. Así como otro grupo significativo de relaciones de estos, con otros países del primer mundo: Alemania, Gran Bretaña, Rusia, España, Italia, etc. Sin embargo, no es significativa la relación de colaboración que existe entre los propios países latinoamericanos, donde incluso algunos sólo muestran relaciones exclusivas con EE.UU, y, en general, México y Brasil son los países dentro de los más productores de tecnologías en $\mathrm{AL}$, que a su vez, muestran un mayor número de relaciones de colaboración e intercambio con otros países de la región para generar nuevos conocimientos tecnológicos.

\section{Análisis de los principales resultados}

La producción tecnológica de AL registrada en la base de datos de patentes concedidas de EE.UU. asciende después de su normalización a 1482 patentes entre septiembre del año 1996 y abril del 2007. Siendo Brasil el país con mayor productividad en la región.

Mediante la aplicación de los indicadores propuestos, se obtuvo información relevante sobre diferentes aspectos. Por ejemplo, solo existen 8 países con titulares con más de cuatro registros de invención, y ninguno de ellos posee más de 4 patentes registradas en otros países de AL. Lo que refleja, entre otras interpretaciones, una regionalización de las producciones tecnológicas y las innovaciones, así como la comercialización de las tecnologías a mercados muy específicos.

Otro resultado significativo del análisis fue conocer que en la producción tecnológica de AL solo existe un núcleo reducido de titulares con una mayor productividad. Mediante los indicadores propuestos se identificaron de ellos los principales signatarios con el mayor liderazgo tecnológico de la región, excepto Brasil y México que tienen la mayor cantidad de titulares con más de 4 patentes concedidas. El resto de los titulares por países tienen una producción discreta. Por ejemplo, Venezuela ha logrado ocupar el tercer lugar por países, con dos titulares generando y comercializando intensivamente sus tecnologías en el mercado internacional.

Del análisis de este indicador, en primera instancia, se infiere una poca actividad innovadora y hasta de transferencia y comercialización de tecnología entre los propios países de la región, sus tecnólogos y titulares. Y del análisis de los resultados por titulares se conocen las verdaderas fortalezas de la industria de cada país, entre otros aspectos.

Los análisis por los diferentes niveles de la CIP permitieron estructurar la producción tecnológica de AL según las áreas reconocidas de la técnica. En este caso, se observa que la estructura por secciones no se corresponde a la distribución por clases. El mayor número de patentes pertenece a la sección C (Química), mientras el mayor número de patentes por clases corresponde a la sección A (Necesidades corrientes de la vida). De lo que se infiere que existe un mayor nivel de aplicación 
en las invenciones correspondientes a la clase A61, existiendo un mayor número de innovaciones relacionadas con éste área del conocimiento. A partir de este tipo de análisis se puede disponer de un acercamiento a la estructura del conocimiento tecnológico, por áreas de aplicación en AL. Infiriendo, en este caso, que las mayores fortalezas investigadoras e innovadoras de la región están en el área temática de las Ciencias Médicas o Veterinarias, e Higiene, a pesar de tener un mayor número de patentes en la sección técnica de Química, Metalúrgica.

La producción tecnológica de los titulares por la CIP, permitió representar la taxonomía del conocimiento tecnológico de las industrias, firmas, empresas, etc de AL. Los autores consideran especialmente significativos los análisis generados de la aplicación de este indicador para estudiar la transdisciplinariedad de las tecnologías mediante las producciones de las industrias, las innovaciones protegidas en las empresas, las alianzas de las universidades, etc.

Los indicadores relacionados con el análisis de las clasificaciones conjuntas por subclases temáticas, es otro resultado importante de esta investigación, que permite profundizar en la caracterización por áreas temáticas de la producción tecnológica. La Figura 2 es un ejemplo de cómo se puede representar las estructuras de relaciones de conocimiento tecnológico donde se pueden descifrar tanto relaciones inter disciplinares como transdisciplinares.

\section{Conclusiones}

La investigación cumple con el objetivo planteado al lograr representar la producción tecnológica de AL, profundizando en la producción de Brasil dentro del estudio por países, utilizando para la representación de la producción de patentes, los indicadores simples y relacionales propuestos en esta investigación, confirmados mediante el estudio de caso. Constituyendo los indicadores propuestos medidas validas, confiables y verificables para otros estudios de análisis de la información contenida en los documentos de patentes.

\section{Referencias}

Albuquerque, E.; Simões, R.; Baessa, A.; Campolina, B., y Silva L. (2001): A distribuição espacial da produção científica e tecnológica brasileira: uma descrição de estatísticas de produção local de patentes e artigos científicos. Disponible en: http://www.anpec. org.br/encontro2001/artigos/200105365.pdf [consultado: 12/7/2008].

Alfaraz, P. H., y Calviño, A. M. (2004): Bibliometric study on food science and technology: scientific production in Iberian-American countries (1991-2000): Scientometrics, vol. $61, n^{\circ} 1,89-102$.

Antunes M. S.; Souza M. G. de, y Dutra E. D. (2001): Desarrollo de la tecnología en la industria química de Brasil y Venezuela: Énfasis en patentes. En Revista Espacios, vol. 22 (2). Disponible en http://www.revistaespacios.com/a01v22n02/31012202.html [consultado: 4/5/2008]. 
Arenas, M.; Dovalina, M. P., y Licea de Arenas, J. (2004): La investigación agrícola en América latina y el Caribe desde una perspectiva bibliométrica. Anales de Documentación, $\mathrm{n}^{\circ}$ 7, 29-38.

Arundel, A. (2000): Patents -the Viagra of Innovation Policy. Internal Report to the Expert Group. MERIT.

Corder S. (2006): Políticas de inovação tecnológica no Brasil: experiência recente e perspectivas. (Texto para discusión No 1244) Brasilia, deciembre de 2006. Disponible en: http://www.ipea.gov.br/sites/000/2/publicacoes/tds/td1244.pdf [consultado: $15 / 12 / 2007]$.

Díaz, M. (2005): La información de patentes en el ciclo de vida de un proyecto de investigación: caso de estudio [Tesis en opción al título de Master en Ciencias de la Información] Facultad Comunicación e Información Científica, La Habana.

Díaz, M. (2007): Producción tecnológica de América Latina con mayor visibilidad internacional: 1996- 2007. [Tesina en opción al Diploma de Estudios Avanzados] Universidad de Granada, España.

Edsberg R.; Truffer I., y Raimondo E. (2000): Los indicadores de patentes en Iberoamérica (1990-2000) Disponible en: http://www.ricyt.org/interior/difusion/pubs/elc/9.pdf [consultado: 22/1/2008].

Fernández, M. T.; Sancho, R.; Morillo, F.; Filippo, D., y Gómez, I. (2004): Indicadores de especialización temática de los países de América Latina. [En línea]. In: Taller de Indicadores de Ciencia y Tecnología Iberoamericano e Intermericano. (6.. : Buenos Aires: 2004). [Memorias]. Disponible en: http://www.ricyt.org/interior/normalizacion/VItaller/ S5_produc/fernandezdoc.pdf [consultado el 22 de marzo de 2005].

Ferreira, H. (2007): Da patenteabilidade de elementos do patrimônio genético. [Tesis en opción al título de Master en Dirección] Pontifica Universidad Católica de Sao Pablo. Brasil. Disponible en: http://www.sapientia.pucsp.br//tde_busca/arquivo. php?codArquivo=5892 [consultado: 14/4/2008].

Herrero-Solana, V., y Ríos-Gómez, C. (2006): Producción latinoamericana en biblioteconomía y documentación en el Social Science Citation Index (SSCI) 1966-2003. Information Research, vol. 11, n. ${ }^{\circ} 2$.

Luís-Pinto, A.; Moreiro, J. A. (2005): Búsqueda de salida al estancamiento tecnológico de las universidades: el problema de la visibilidad innovadora en Brasil. En Revista Interamericana de Bibliotecología, Medellín, v. 27, n. ${ }^{2}$, pp. 71-90, jul./dic 2005. Disponible en http://adilsonluiz.files.wordpress.com/2007/07/reviberbib.pdf [consultado: 25/5/2008].

Luna, F.; Baessa, A., y Franco, P. (2007): Impacto das marcas e patentes no desempenho econômico das firmas. Disponible en: http://www.anpec.org.br/encontro2007/artigos/ A07A155.pdf [consultado: 25/12/2007].

Matias-Pereira, J. (2004): Política Industrial, Propiedad Intelectual y Desarrollo en América Latina. En Contribuciones a la Economía. Disponible en: http://www.eumed.net/ ce/2004/jmp-al.htm [consultado: 2/3/2008].

Michel, J., y Bernd, B. (2001): Patent citation analysis a closer look at the basic input data from patent search reports. Scientometrics, 51 (1):185-201.

Morgado, A. P. (2005): Inovação tecnologica no Brasil: uma análise das indústriais brasileiras através da pesquisa de inovação tecnológica (PINTEC). [Tesis en opción al tí- 
tulo de Master en Sistemas de Gestión]. Universidad Federal Fluminense, Brasil. Disponible en: http://www.bdtd.ndc.uff.br/tde_busca/arquivo.php?codArquivo=1257 [consultado: 14/4/2008].

Moya-Anegón, F., y Herrero-Solana, V. (2002): Visibilidad internacional de la producción científica iberoamericana en biblioteconomía y documentación (2001-2000), Ciência da Informação, Brasilia, vol. 31, n 3, 54-65.

Moya-Anegón, F., y Herrero-Solana, V. (1999): Science in America Latina: a comparison of bibliometric and scientific technical indicators. Scientometrics, vol. 46, $\mathrm{n}^{\circ}$ 2, 299-320.

Moya-Anegón, F, y Díaz, M. (2008): El análisis de patentes como estrategia para la toma de decisiones innovadoras. En El profesional de la información, mayo-junio, vol. 17, $\mathrm{n}^{\circ}$ 3, 293-302. Disponible en: http://elprofesionaldelainformacion.metapress.com/me$\mathrm{dia} / \mathrm{hpb} 9 j d h y w q 1 q n l d t w c 2 l / c o n t r i b u t i o n s / y / 7 / 6 / 1 / y 761611025 \mathrm{k} 32072 . p d f$ [consultado: 2/6/2008].

Narin, F., y Hamilton, K. S. (1996): Bibliometric performance measures. Scientometrics, vol. 36, no 3, 293-310.

Narin, F.; Kimberly, S., y Dominic, O. (1997): The increasing linkage between U.S. technology and public science. Research Policy: 317-330.

Narvaez-Berthelemot, N. (1995): An index to measure the international collaboration of developing countries based on the participation of national institutions: the case of Latin America. Scientometrics, vol. 34, $\mathrm{n}^{\circ}$ 1, 37-55.

Narvaez-Berthelemot, N.; Almada de Ascencio, M., y Russell, J. M. (1993): International scientific collaboration: cooperation between Latin America and Spain, as seen from different databases. Journal of Information Science, vol. 19, pp. 389-394.

Pavitt, K. (1988): Uses and abuses of patent statistics, En Van Raan, A. F. J. (ed.), Handbook of Quantitative Studies of Science and Technology. Elsevier Science Publishers B.V. North Holland.

Plaza, L. M., y Albert, A. (2001): La ciencia básica al servicio del desarrollo tecnológico. Principales indicadores para países de América Latina. V Taller Iberoamericano Interamericano de Indicadores de Ciencia y Tecnología. 15-18 octubre, 2001. Montevideo. Uruguay. www.ricyt.edu.ar/interior/normalizacion/V_taller/plaza [consultado en enero de 2007].

Rovira, J. (2008): Innovación y acceso a los medicamentos: contradicciones y propuestas. En: Revista Economía de la salud, vol. 6 (4). Disponible en http://www.economiadelasalud.com/Ediciones/64/08_pdf/Innovacion.pdf [consultado: 5/5/2008].

Russell, J. M.; Correa, M. S.; García, N.; Guadarrama, J. A., y Priego, L. (1987): Research and publication trends of a Latin American veterinary faculty. Interciencia, vol. 12, $\mathrm{n}^{\circ} 5,243-244$.

RusselL, J. M., y Narvaez-Berthelemot, N. (1992): Use of the bibliometrical method to determine the scientific activity of Latin America: The case of international cooperation. International Journal of Information and Library Research, vol. 4, 127-137.

Saavedra, O.; Sotolongo, G., y Guzmán, M. V. (2002): Medición de la producción científica en América Latina y el Caribe en el campo agrícola y afines: un estudio bibliométrico. Revista Española de Documentación Científica, vol. 25, pp. 151-161.

Sánchez M.; Cano V., y Esparza E. (s.a): Un análisis de las patentes como indicadores. Algunas consideraciones conceptuales. Universidad de La Laguna Enrique Los Arcos 
y Oficina Europea de Patentes. Disponible en: http://www.ucm.es/info/ec/jec9/pdf/ A11\%20-\%20S\%e1nchez\%20Padr\%f3n,\%20Miguel,\%20Cano,\%20Victor,\%20Esparza, $\% 20$ Encarn aci\%f3n,\%20Los\%20Arcos,\%20Enrique.pdf [consultado en noviembre de 2009].

Sánchez, J. M.; Medina, J. R., y León, A. M. (2007): Publicación internacional de patentes por organizaciones e inventores de origen colombiano. En Cuadernos de Economía, v. XXVI, n. 47, 247-270. Disponible en http://www.fce.unal.edu.co/cuadernos/47/9PUBLICACIONES.pdf [consultado: 10/5/2008].

Sancho, R.; Morillo, F.; Filippo, D.; Gómez, I., y Fernández, M. T. (2006): Indicadores de colaboración científica intercentros en los países de América Latina. Interciencia, vol. 31 (4), 284-292.

Sikasso, L. (2007): Caracterização da proteção ás patentes como estímulo do desenvolvimiento econômico. [Tesis en opción al título de Master en Dirección]. Universidad Católica de Brasilia. Brasil. Disponible en: http://bdtd.ufrrj.br//tde_busca/arquivo. php?codArquivo=283 [consultado: 14/4/2008].

Trilateral Co-operation, (2005) Trilateral Statistical Report 2004. Edition Munich, Germany. [Web Page] Disponible en: http://www.trilateral.net/tsr/tsr_2004/ch2/ [consultado en septiembre de 2006].

\section{ANEXOS}

\section{Anexo 1. Códigos de países de América Latina seleccionados para el estudio}
1. (AR) Argentina
2. (BO) Bolivia
3. (BR) Brasil
4. (CL) Chile
5. (CO) Colombia
6. (CR) Costa Rica
7. (CU) Cuba
8. (DO) República Dominicana
9. (EC) Ecuador
10. (SV) El Salvador
11. (GT) Guatemala
12. (HT) Haití
13. (HN) Honduras
14. (MQ) Martinica
15. (MX) México
16. (NI) Nicaragua
17. (PA) Panamá
18. (PY) Paraguay
19. (PE) Perú
20. (UY)Uruguay
21. (VE) Venezuela 


\section{Anexo 2. Secciones de la Clasificación Internacional de Patentes (CIP)}

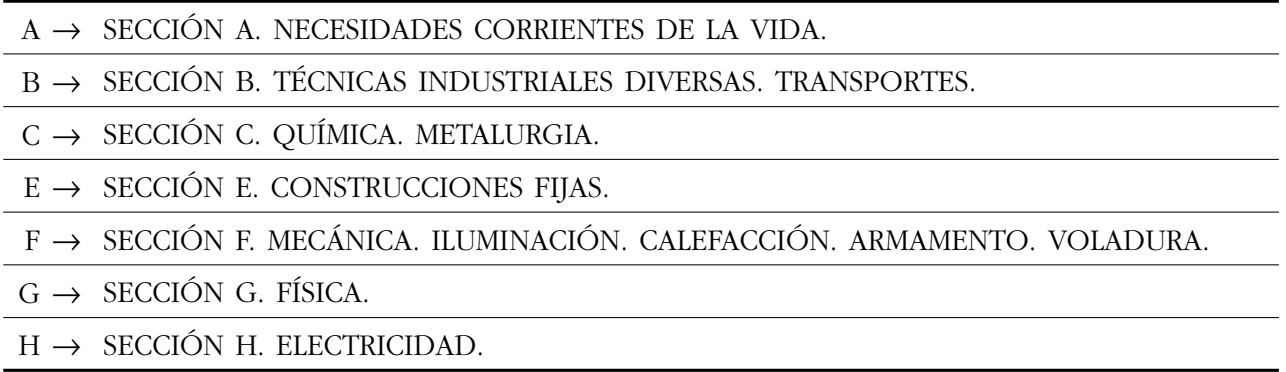

\section{Anexo 3. Subespecialidades temáticas de la CIP}

\begin{tabular}{|c|c|c|}
\hline \multirow[t]{3}{*}{$\mathrm{A} 1 \mathrm{H} \rightarrow$} & A & SECCIÓN A. NECESIDADES CORRIENTES DE LA VIDA. \\
\hline & 1 & AGRICULTURA. SILVICULTURA. CRÍA. CAZA. CAPTURA. PESCA. \\
\hline & $\mathrm{H}$ & $\begin{array}{l}\text { NOVEDADES VEGETALES O PROCEDIMIENTOS PARA SU OBTENCIÓN. REPRODUC- } \\
\text { CIÓN DE PLANTAS POR TÉCNICAS DE CULTIVO DE TEJIDOS. }\end{array}$ \\
\hline \multirow[t]{3}{*}{$\mathrm{A} 61 \mathrm{~B} \rightarrow$} & A & SECCIÓN A. NECESIDADES CORRIENTES DE LA VIDA. \\
\hline & 61 & CIENCIAS MÉDICAS O VETERINARIAS. HIGIENE. \\
\hline & $\mathrm{B}$ & DIAGNÓSTICO. CIRUGÍA. IDENTIFICACIÓN. \\
\hline \multirow[t]{3}{*}{$\mathrm{A} 61 \mathrm{~F} \rightarrow$} & A & SECCIÓN A. NECESIDADES CORRIENTES DE LA VIDA. \\
\hline & 61 & CIENCIAS MÉDICAS O VETERINARIAS. HIGIENE. \\
\hline & $\mathrm{F}$ & $\begin{array}{l}\text { FILTROS IMPLANTABLES EN LOS VASOS SANGUÍNEOS. PRÓTESIS. DISPOSITIVOS } \\
\text { QUE MANTIENEN LA LUZ O QUE EVITAN EL COLAPSO DE ESTRUCTURAS TUBU- } \\
\text { LARES, p. ej. STENTS. DISPOSITIVOS DE ORTOPEDIA, CURA O PARA LA CONTRA- } \\
\text { CEPCIÓN. FOMENTACIÓN. TRATAMIENTO O PROTECCIÓN DE OJOS Y OÍDOS. } \\
\text { VENDAJES, APÓSITOS O COMPRESAS ABSORBENTES. BOTIQUINES DE PRIMEROS } \\
\text { AUXILIOS. }\end{array}$ \\
\hline \multirow[t]{3}{*}{$\mathrm{A} 61 \mathrm{~K} \rightarrow$} & A & SECCIÓN A. NECESIDADES CORRIENTES DE LA VIDA. \\
\hline & 61 & CIENCIAS MÉDICAS O VETERINARIAS. HIGIENE. \\
\hline & $\mathrm{K}$ & PREPARACIONES DE USO MÉDICO, DENTAL O PARA EL ASEO. \\
\hline \multirow[t]{3}{*}{$\mathrm{B} 1 \mathrm{D} \rightarrow$} & $\mathrm{B}$ & SECCIÓN B. TÉCNICAS INDUSTRIALES DIVERSAS. TRANSPORTES. \\
\hline & 1 & PROCEDIMIENTOS O APARATOS FÍSICOS O QUÍMICOS EN GENERAL. \\
\hline & $\mathrm{D}$ & SEPARACIÓN. \\
\hline \multirow[t]{3}{*}{$\mathrm{B} 1 \mathrm{~J} \rightarrow$} & $\mathrm{B}$ & SECCIÓN B. TÉCNICAS INDUSTRIALES DIVERSAS. TRANSPORTES. \\
\hline & 1 & PROCEDIMIENTOS O APARATOS FÍSICOS O QUÍMICOS EN GENERAL. \\
\hline & $\mathrm{J}$ & $\begin{array}{l}\text { PROCEDIMIENTOS QUÍMICOS O FÍSICOS, p. ej. CATÁLISIS, QUÍMICA DE LOS CO- } \\
\text { LOIDES. APARATOS ADECUADOS. }\end{array}$ \\
\hline
\end{tabular}




\begin{tabular}{|c|c|c|}
\hline \multirow[t]{3}{*}{$\mathrm{B} 21 \mathrm{D} \rightarrow$} & $\mathrm{B}$ & SECCIÓN B. TÉCNICAS INDUSTRIALES DIVERSAS. TRANSPORTES. \\
\hline & 21 & $\begin{array}{l}\text { TRABAJO MECÁNICO DE LOS METALES SIN ARRANQUE SUSTANCIAL DE MATERIAL. } \\
\text { CORTE DEL METAL POR PUNZONADO. }\end{array}$ \\
\hline & $\mathrm{D}$ & $\begin{array}{l}\text { TRABAJO MECÁNICO O TRATAMIENTO DE CHAPAS, TUBOS, BARRAS O PERFILES } \\
\text { METÁLICOS SIN ARRANQUE SUSTANCIAL DE MATERIAL. CORTE POR PUNZONADO. }\end{array}$ \\
\hline \multirow[t]{3}{*}{$\mathrm{B} 29 \mathrm{C} \rightarrow$} & $\mathrm{B}$ & SECCIÓN B. TÉCNICAS INDUSTRIALES DIVERSAS. TRANSPORTES. \\
\hline & 29 & $\begin{array}{l}\text { TRABAJO DE LAS MATERIAS PLÁSTICAS. TRABAJO DE SUSTANCIAS EN ESTADO } \\
\text { PLÁSTICO EN GENERAL. }\end{array}$ \\
\hline & $\mathrm{C}$ & $\begin{array}{l}\text { CONFORMACIÓN O UNIÓN DE LAS MATERIAS PLÁSTICAS. CONFORMACIÓN O } \\
\text { UNIÓN DE SUSTANCIAS EN ESTADO PLÁSTICO EN GENERAL. POSTRATAMIENTO } \\
\text { DE PRODUCTOS CONFORMADOS, p. ej. REPARACIÓN. }\end{array}$ \\
\hline \multirow[t]{3}{*}{$\mathrm{B} 32 \mathrm{~B} \rightarrow$} & $\mathrm{B}$ & SECCIÓN B. TÉCNICAS INDUSTRIALES DIVERSAS. TRANSPORTES. \\
\hline & 32 & PRODUCTOS ESTRATIFICADOS. \\
\hline & $\mathrm{B}$ & $\begin{array}{l}\text { PRODUCTOS ESTRATIFICADOS, es decir, HECHOS DE VARIAS CAPAS DE FORMA } \\
\text { PLANA O NO PLANA, p. ej. CELULAR O EN NIDO DE ABEJA. }\end{array}$ \\
\hline \multirow[t]{3}{*}{$\mathrm{B} 63 \mathrm{~B} \rightarrow$} & $\mathrm{B}$ & SECCIÓN B. TÉCNICAS INDUSTRIALES DIVERSAS. TRANSPORTES. \\
\hline & 63 & NAVÍOS U OTRAS EMBARCACIONES FLOTANTES. SUS EQUIPOS. \\
\hline & $\mathrm{B}$ & NAVÍOS U OTRAS EMBARCACIONES FLOTANTES. MATERIAL DE ARMAMENTO. \\
\hline \multirow[t]{3}{*}{$\mathrm{B} 65 \mathrm{D} \rightarrow$} & B & SECCIÓN B. TÉCNICAS INDUSTRIALES DIVERSAS. TRANSPORTES. \\
\hline & 65 & $\begin{array}{l}\text { TRANSPORTE. EMBALAJE. ALMACENADO. MANIPULACIÓN DE MATERIALES DELGA- } \\
\text { DOS O FILIFORMES. }\end{array}$ \\
\hline & $\mathrm{D}$ & $\begin{array}{l}\text { RECEPTÁCULOS PARA EL ALMACENAMIENTO O EL TRANSPORTE DE OBJETOS O } \\
\text { MATERIALES, p. ej. SACOS, BARRILES, BOTELLAS, CAJAS, LATAS, CARTONES, ARCAS, } \\
\text { BOTES, BIDONES, TARROS, TANQUES. ACCESORIOS O CIERRES PARA RECEPTÁCU- } \\
\text { LOS. ELEMENTOS DE EMBALAJE. PAQUETES. }\end{array}$ \\
\hline \multirow[t]{3}{*}{$\mathrm{B} 8 \mathrm{~B} \rightarrow$} & B & SECCIÓN B. TÉCNICAS INDUSTRIALES DIVERSAS. TRANSPORTES. \\
\hline & 8 & LIMPIEZA. \\
\hline & $\mathrm{B}$ & LIMPIEZA EN GENERAL. PREVENCIÓN DE LA SUCIEDAD EN GENERAL. \\
\hline \multirow[t]{3}{*}{ C10G $\rightarrow$} & $\mathrm{C}$ & SECCIÓN C. QUÍMICA. METALURGIA. \\
\hline & 10 & $\begin{array}{l}\text { INDUSTRIAS DEL PETRÓLEO, GAS O COQUE. GAS DE SÍNTESIS QUE CONTIENE } \\
\text { MONÓXIDO DE CARBONO. COMBUSTIBLES. LUBRICANTES. TURBA. }\end{array}$ \\
\hline & G & $\begin{array}{l}\text { CRACKING DE LOS ACEITES DE HIDROCARBUROS. PRODUCCIÓN DE MEZCLAS DE } \\
\text { HIDROCARBUROS LIQUIDOS, p. ej. POR HIDROGENACIÓN DESTRUCTIVA, POR } \\
\text { OLIGOMERIZACIÓN, POR POLIMERIZACIÓN. RECUPERACIÓN DE ACEITES DE HI- } \\
\text { DROCARBUROS A PARTIR DE ESQUISTOS, DE ARENA PETROLÍFERA O GASES. RE- } \\
\text { FINO DE MEZCLAS COMPUESTAS PRINCIPALMENTE DE HIDROCARBUROS. REFOR- } \\
\text { MADO DE NAFTA. CERAS MINERALES. }\end{array}$ \\
\hline
\end{tabular}




\begin{tabular}{|c|c|c|}
\hline \multirow[t]{3}{*}{$\mathrm{C} 10 \mathrm{~L} \rightarrow$} & $\mathrm{C}$ & SECCIÓN C. QUÍMICA. METALURGIA. \\
\hline & 10 & $\begin{array}{l}\text { INDUSTRIAS DEL PETRÓLEO, GAS O COQUE. GAS DE SÍNTESIS QUE CONTIENE } \\
\text { MONÓXIDO DE CARBONO. COMBUSTIBLES. LUBRICANTES. TURBA. }\end{array}$ \\
\hline & $\mathrm{L}$ & $\begin{array}{l}\text { COMBUSTIBLES NO PREVISTOS EN OTROS LUGARES. GAS NATURAL. GAS NATURAL } \\
\text { DE SÍNTESIS OBTENIDO POR PROCEDIMIENTOS NO PREVISTOS EN LAS SUBCLASES, } \\
\text { GAS DE PETRÓLEO LICUADO. ADICIÓN DE SUSTANCIAS A LOS COMBUSTIBLES O } \\
\text { AL FUEGO PARA REDUCIR EL HUMO O DEPÓSITOS INDESEABLES, O PARA FACILI- } \\
\text { TAR LA ELIMINACIÓN DEL HOLLÍN. GENERADORES DE FUEGO. }\end{array}$ \\
\hline \multirow[t]{3}{*}{$\mathrm{C} 12 \mathrm{~N} \rightarrow$} & $\mathrm{C}$ & SECCIÓN C. QUÍMICA. METALURGIA. \\
\hline & 12 & $\begin{array}{l}\text { BIOQUÍMICA. CERVEZA. BEBIDAS ALCOHÓLICAS. VINO. VINAGRE. MICROBIOLO- } \\
\text { GÍA. ENZIMOLOGÍA. TÉCNICAS DE MUTACIÓN O DE GENÉTICA. }\end{array}$ \\
\hline & $\mathrm{N}$ & $\begin{array}{l}\text { MICROORGANISMOS O ENZIMAS. COMPOSICIONES QUE LOS CONTIENEN. CULTI- } \\
\text { VO O CONSERVACIÓN DE MICROORGANISMOS. TÉCNICAS DE MUTACIÓN O DE } \\
\text { INGENIERÍA GENÉTICA. MEDIOS DE CULTIVO. }\end{array}$ \\
\hline \multirow[t]{3}{*}{$\mathrm{C} 12 \mathrm{P} \rightarrow$} & $\mathrm{C}$ & SECCIÓN C. QUÍMICA. METALURGIA. \\
\hline & 12 & $\begin{array}{l}\text { BIOQUÍMICA. CERVEZA. BEBIDAS ALCÓHOLICAS. VINO. VINAGRE. MICROBIOLO- } \\
\text { GÍA. ENZIMOLOGÍA. TÉCNICAS DE MUTACIÓN O DE GENÉTICA. }\end{array}$ \\
\hline & $\mathrm{P}$ & $\begin{array}{l}\text { PROCESOS DE FERMENTACIÓN O PROCESOS QUE UTILIZAN ENZIMAS PARA LA } \\
\text { SÍNTESIS DE UN COMPUESTO QUÍMICO DADO O DE UNA COMPOSICIÓN DADA, } \\
\text { O PARA LA SEPARACIÓN DE ISOMEROS ÓPTICOS A PARTIR DE UNA MEZCLA RA- } \\
\text { CÉMICA. }\end{array}$ \\
\hline \multirow[t]{3}{*}{$\mathrm{C} 1 \mathrm{~B} \rightarrow$} & $\mathrm{C}$ & SECCIÓN C. QUÍMICA. METALURGIA. \\
\hline & 1 & QUÍMICA INORGÁNICA. \\
\hline & $\mathrm{B}$ & ELEMENTOS NO METÁLICOS. SUS COMPUESTOS. \\
\hline \multirow[t]{3}{*}{$\mathrm{C} 21 \mathrm{~B} \rightarrow$} & $\mathrm{C}$ & SECCIÓN C. QUÍMICA. METALURGIA. \\
\hline & 21 & METALURGIA DEL HIERRO. \\
\hline & $\mathrm{B}$ & FABRICACIÓN DEL HIERRO O DEL ACERO. \\
\hline \multirow[t]{3}{*}{$\mathrm{C} 4 \mathrm{~B} \rightarrow$} & $\mathrm{C}$ & SECCIÓN C. QUÍMICA. METALURGIA. \\
\hline & 4 & CEMENTOS. HORMIGÓN. PIEDRA ARTIFICIAL. CERÁMICAS. REFRACTARIOS. \\
\hline & $\mathrm{B}$ & $\begin{array}{l}\text { CAL. MAGNESIA. ESCORIAS. CEMENTOS. SUS COMPOSICIONES, p. ej. MORTEROS, } \\
\text { HORMIGÓN O MATERIALES DE CONSTRUCCIÓN SIMILARES. PIEDRA ARTIFICIAL. } \\
\text { CERÁMICAS. REFRACTARIOS. TRATAMIENTO DE LA PIEDRA NATURAL. }\end{array}$ \\
\hline \multirow[t]{3}{*}{$\mathrm{C} 7 \mathrm{C} \rightarrow$} & $\mathrm{C}$ & SECCIÓN C. QUÍMICA. METALURGIA. \\
\hline & 7 & QUÍMICA ORGÁNICA. \\
\hline & $\mathrm{C}$ & COMPUESTOS ACÍCLICOS O CARBOCÍCLICOS. \\
\hline
\end{tabular}




\begin{tabular}{|c|c|c|}
\hline \multirow[t]{3}{*}{$\mathrm{C} 7 \mathrm{H} \rightarrow$} & $\mathrm{C}$ & SECCIÓN C. QUÍMICA. METALURGIA. \\
\hline & 7 & QUÍMICA ORGÁNICA. \\
\hline & $\mathrm{H}$ & AZÚCARES. SUS DERIVADOS. NUCLEÓSIDOS. NUCLEÓTIDOS. ÁCIDOS NUCLÉICOS. \\
\hline \multirow{3}{*}{$\mathrm{C} 7 \mathrm{~K} \rightarrow$} & $\mathrm{C}$ & SECCIÓN C. QUÚMICA. METALURGIA. \\
\hline & 7 & QUÍMICA ORGÁNICA. \\
\hline & $\mathrm{K}$ & PÉPTIDOS. \\
\hline \multirow[t]{3}{*}{$\mathrm{C} 8 \mathrm{~F} \rightarrow$} & $\mathrm{C}$ & SECCIÓN C QUÍMICA. METALURGIA. \\
\hline & 8 & $\begin{array}{l}\text { COMPUESTOS MACROMOLECULARES ORGÁNICOS. SU PREPARACIÓN O PRODUC- } \\
\text { CIÓN QUÍMICA. COMPOSICIONES BASADAS EN COMPUESTOS MACROMOLECULA- } \\
\text { RES. }\end{array}$ \\
\hline & $\mathrm{F}$ & $\begin{array}{l}\text { COMPUESTOS MACROMOLECULARES OBTENIDOS POR REACCIONES QUE IMPLICAN } \\
\text { ÚNICAMENTE ENLACES INSATURADOS CARBONO - CARBONO. }\end{array}$ \\
\hline \multirow[t]{3}{*}{$\mathrm{C} 8 \mathrm{~K} \rightarrow$} & $\mathrm{C}$ & SECCIÓN C. QUÍMICA. METALURGIA. \\
\hline & 8 & $\begin{array}{l}\text { COMPUESTOS MACROMOLECULARES ORGÁNICOS. SU PREPARACIÓN O PRODUC- } \\
\text { CIÓN QUÍMICA. COMPOSICIONES BASADAS EN COMPUESTOS MACROMOLECULA- } \\
\text { RES. }\end{array}$ \\
\hline & $\mathrm{K}$ & $\begin{array}{l}\text { UTILIZACIÓN DE SUSTANCIAS INORGÁNICAS U ORGÁNICAS NO MACROMOLECU- } \\
\text { LARES COMO INGREDIENTES DE LA COMPOSICIÓN. }\end{array}$ \\
\hline \multirow[t]{3}{*}{$\mathrm{D} 21 \mathrm{~F} \rightarrow$} & $\mathrm{D}$ & SECCIÓN D. TEXTILES. PAPEL. \\
\hline & 21 & FABRICACIÓN DEL PAPEL. PRODUCCIÓN DE LA CELULOSA. \\
\hline & $\mathrm{F}$ & MÁQUINAS DE FABRICAR PAPEL. MÉTODOS DE PRODUCCIÓN DEL PAPEL. \\
\hline \multirow[t]{3}{*}{$\mathrm{E} 21 \mathrm{~B} \rightarrow$} & $\mathrm{E}$ & SECCIÓN E. CONSTRUCCIONES FIJAS. \\
\hline & 21 & PERFORACIÓN DEL SUELO O DE LA ROCA. EXPLOTACIÓN MINERA. \\
\hline & $\mathrm{B}$ & $\begin{array}{l}\text { PERFORACIÓN DEL SUELO O DE LA ROCA. EXTRACCIÓN DE PETRÓLEO, GAS, AGUA } \\
\text { O MATERIALES SOLUBLES O FUNDIBLES O DE UNA SUSPENSIÓN DE MATERIAS } \\
\text { MINERALES A PARTIR DE POZOS. }\end{array}$ \\
\hline \multirow[t]{3}{*}{$\mathrm{E} 2 \mathrm{D} \rightarrow$} & $\mathrm{E}$ & SECCIÓN E. CONSTRUCCIONES FIJAS. \\
\hline & 2 & HIDRÁULICA. CIMENTACIONES. MOVIMIENTO DE TIERRAS. \\
\hline & $\mathrm{D}$ & $\begin{array}{l}\text { CIMENTACIONES. EXCAVACIONES. TERRAPLENES. OBRAS SUBTERRÁNEAS O BAJO } \\
\text { EL AGUA. }\end{array}$ \\
\hline \multirow[t]{3}{*}{$\mathrm{F} 16 \mathrm{~J} \rightarrow$} & $\mathrm{F}$ & SECCIÓN F. MECÁNICA. ILUMINACIÓN. CALEFACCIÓN. ARMAMENTO. VOLADURA. \\
\hline & 16 & $\begin{array}{l}\text { ELEMENTOS O CONJUNTOS DE TECNOLOGÍA. MEDIDAS GENERALES PARA ASEGU- } \\
\text { RAR EL BUEN FUNCIONAMIENTO DE LAS MÁQUINAS O INSTALACIONES. AISLA- } \\
\text { MIENTO TÉRMICO EN GENERAL. }\end{array}$ \\
\hline & $\mathrm{J}$ & $\begin{array}{l}\text { PISTONES. CILINDROS. RECIPIENTES A PRESIÓN EN GENERAL. JUNTAS DE ESTAN- } \\
\text { QUEIDAD. }\end{array}$ \\
\hline
\end{tabular}


Producción tecnológica latinoamericana con mayor visibilidad internacional: 1996-2007

\begin{tabular}{|c|c|c|}
\hline \multirow[t]{3}{*}{$\mathrm{F} 16 \mathrm{~L} \rightarrow$} & $\mathrm{F}$ & SECCIÓN F. MECÁNICA. ILUMINACIÓN. CALEFACCIÓN. ARMAMENTO. VOLADURA. \\
\hline & 16 & $\begin{array}{l}\text { ELEMENTOS O CONJUNTOS DE TECNOLOGÍA. MEDIDAS GENERALES PARA ASEGU- } \\
\text { RAR EL BUEN FUNCIONAMIENTO DE LAS MÁQUINAS O INSTALACIONES. AISLA- } \\
\text { MIENTO TÉRMICO EN GENERAL. }\end{array}$ \\
\hline & $\mathrm{L}$ & $\begin{array}{l}\text { TUBERÍAS O TUBOS. EMPALMES U OTROS ACCESORIOS PARA TUBERÍAS. SOPORTES } \\
\text { PARA TUBOS, CABLES O CONDUCTOS PROTECTORES. MEDIOS DE AISLAMIENTO } \\
\text { TÉRMICO EN GENERAL. }\end{array}$ \\
\hline \multirow[t]{3}{*}{$\mathrm{F} 1 \mathrm{P} \rightarrow$} & $\mathrm{F}$ & SECCIÓN F. MECÁNICA. ILUMINACIÓN. CALEFACCIÓN. ARMAMENTO. VOLADURA. \\
\hline & 1 & $\begin{array}{l}\text { MÁQUINAS O MOTORES EN GENERAL. PLANTAS MOTRICES EN GENERAL. MÁQUI- } \\
\text { NAS DE VAPOR. }\end{array}$ \\
\hline & $\mathrm{P}$ & $\begin{array}{l}\text { REFRIGERACIÓN DE MÁQUINAS O MOTORES EN GENERAL. REFRIGERACIÓN DE } \\
\text { MOTORES DE COMBUSTIÓN INTERNA. }\end{array}$ \\
\hline \multirow[t]{3}{*}{$\mathrm{F} 25 \mathrm{~B} \rightarrow$} & $\mathrm{F}$ & SECCIÓN F. MECÁNICA. ILUMINACIÓN. CALEFACCIÓN. ARMAMENTO. VOLADURA. \\
\hline & 25 & $\begin{array}{l}\text { REFRIGERACIÓN O ENFRIAMIENTO. SISTEMAS COMBINADOS DE CALEFACCIÓN Y } \\
\text { DE REFRIGERACIÓN. SISTEMAS DE BOMBA DE CALOR. FABRICACIÓN O ALMACE- } \\
\text { NAMIENTO DEL HIELO. LICUEFACCIÓN O SOLIDIFICACIÓN DE GASES. }\end{array}$ \\
\hline & $\mathrm{B}$ & $\begin{array}{l}\text { MÁQUINAS, INSTALACIONES O SISTEMAS FRIGORÍFICOS. SISTEMAS COMBINADOS } \\
\text { DE CALEFACCIÓN Y DE REFRIGERACIÓN. SISTEMAS DE BOMBA DE CALOR. }\end{array}$ \\
\hline \multirow[t]{3}{*}{$\mathrm{F} 25 \mathrm{D} \rightarrow$} & $\mathrm{F}$ & SECCIÓN F. MECÁNICA. ILUMINACIÓN. CALEFACCIÓN. ARMAMENTO. VOLADURA. \\
\hline & 25 & $\begin{array}{l}\text { REFRIGERACIÓN O ENFRIAMIENTO. SISTEMAS COMBINADOS DE CALEFACCIÓN Y } \\
\text { DE REFRIGERACIÓN. SISTEMAS DE BOMBA DE CALOR. FABRICACIÓN O ALMACE- } \\
\text { NAMIENTO DEL HIELO. LICUEFACCIÓN O SOLIDIFICACIÓN DE GASES. }\end{array}$ \\
\hline & $\mathrm{D}$ & $\begin{array}{l}\text { REFRIGERADORES. CÁMARAS FRÍAS. NEVERAS. APARATOS DE ENFRIAMIENTO O } \\
\text { CONGELACIÓN NO CUBIERTOS POR NINGUNA OTRA SUBCLASE. }\end{array}$ \\
\hline \multirow[t]{3}{*}{$\mathrm{F} 4 \mathrm{~B} \rightarrow$} & $\mathrm{F}$ & SECCIÓN F. MECÁNICA. ILUMINACIÓN. CALEFACCIÓN. ARMAMENTO. VOLADURA. \\
\hline & 4 & $\begin{array}{l}\text { MÁQUINAS DE LIQUIDOS DE DESPLAZAMIENTO POSITIVO. BOMBAS PARA LIQUI- } \\
\text { DOS O PARA FLUIDOS COMPRESIBLES. }\end{array}$ \\
\hline & $\mathrm{B}$ & MÁQUINAS DE LÍQUIDOS DE DESPLAZAMIENTO POSITIVO. BOMBAS. \\
\hline \multirow[t]{3}{*}{$\mathrm{G} 1 \mathrm{~N} \rightarrow$} & G & SECCIÓN G. FÍSICA. \\
\hline & 1 & METROLOGÍA. ENSAYOS. \\
\hline & $\mathrm{N}$ & $\begin{array}{l}\text { INVESTIGACIÓN O ANÁLISIS DE MATERIALES POR DETERMINACIÓN DE SUS PRO- } \\
\text { PIEDADES QUÍMICAS O FÍSICAS. }\end{array}$ \\
\hline \multirow[t]{3}{*}{$\mathrm{G} 2 \mathrm{~B} \rightarrow$} & G & SECCIÓN G. FÍSICA. \\
\hline & 2 & ÓPTICA. \\
\hline & $\mathrm{B}$ & ELEMENTOS, SISTEMAS O APARATOS ÓPTICOS. \\
\hline
\end{tabular}




\begin{tabular}{c|c|l}
\hline \multirow{2}{*}{$\mathrm{H} 1 \mathrm{~B} \rightarrow$} & $\mathrm{H}$ & SECCIÓN H ELECTRICIDAD. \\
\cline { 2 - 3 } & 1 & ELEMENTOS ELÉCTRICOS BÁSICOS. \\
\cline { 2 - 3 } & $\mathrm{B}$ & $\begin{array}{l}\text { CABLES. CONDUCTORES. AISLADORES. EMPLEO DE MATERIALES ESPECIFICOS POR } \\
\text { SUS PROPIEDADES CONDUCTORAS, AISLANTES O DIELÉCTRICAS. }\end{array}$ \\
\hline \multicolumn{3}{|c}{} \\
\hline \multirow{2}{*}{$\mathrm{H} 2 \mathrm{~K} \rightarrow$} & $\mathrm{H}$ & SECCIÓN H ELECTRICIDAD. \\
\cline { 2 - 3 } & 2 & PRODUCCIÓN, CONVERSIÓN O DISTRIBUCIÓN DE LA ENERGÍA ELÉCTRICA. \\
\cline { 2 - 3 } & $\mathrm{K}$ & MÁQUINAS DINAMOELÉCTRICAS. \\
\hline
\end{tabular}

